\title{
Incidencia de los embalses en el régimen térmico del río. El caso del tramo final del río Ebro
}

\author{
Rafael Val ${ }^{1}$. Daniel Niñerola ${ }^{1}$. Joan Armengol ${ }^{2}$. José Dolz ${ }^{1}$ \\ ${ }^{1}$ Departament d'Enginyeria Hidràulica, Marítima i Ambiental, E.T.S.E.C.C.P. Universidad Politécnica de \\ Catalunya. C/J. Girona, 1-3, D1.08034-Barcelona \\ ${ }^{2}$ Departament d'Ecologia. Facultat de Biologia. Universidad de Barcelona. Avd. Diagonal, 645. 08028- \\ Barcelona
}

\begin{abstract}
RESUMEN
El agua del río mantiene un intercambio de calor con la atmósfera y con el fondo; este proceso puede lograr su equilibrio a lo largo de un tramo de río con características geológicas y climáticas similares. Su comportamiento puede ser modificado por efectos artificiales; tales como el uso del agua del río para refrigeración de centrales termoeléctricas o por la existencia de embalses.

En el caso de regiones con clima mediterráneo, donde se encuentran los embalses aquí estudiados, los efectos de las alteraciones en el régimen térmico producido por éstos, provocan una tendencia a la constancia térmica estacional, un aumento de la uniformidad diaria, una elevación de las temperaturas invernales y la disminución de las temperaturas estivales. La temperatura del agua es un factor importante en la ecología del río, ya que condiciona en gran medida la vida de las comunidades fluviales. Aguas abajo de las termoeléctricas o presas, la temperatura del agua evoluciona de tal forma que tiende a lograr el equilibrio con el medio ambiente.
\end{abstract}

Palabras clave: balance térmico, radiación solar, embalse, clima mediterráneo.

\begin{abstract}
The river water maintains a heat exchange with the atmosphere and with the bottom; this process can reach its equilibrium along a stretch of the river with similar geologic and climatic characteristics. Its behavior can be modified by artificial effects, such as the use of the river water for the cooling of thermal power stations or by the presence of reservoirs.

In the case of regions with Mediterranean climate, where the studied reservoirs are located, the effects of changes in the natural thermal regime caused by them produce a trend towards a constant seasonal thermal condition, an increase in daily uniformity, an increase in winter temperatures, and a decrease in summer temperatures. Water temperature is an important factor in the river's ecology, because it greatly conditions the life of fluvial communities. Downstream from power stations or dams, the water temperature evolves in such a way that tends to achieve equilibrium with the environment.
\end{abstract}

Keywords: heat budget, solar radiation, long wave radiation, reservoir, Mediterranean climate.

\section{INTRODUCCIÓN}

Los embalses constituyen una discontinuidad en la dinámica fluvial. Por este motivo, el agua al salir de un embalse presenta características físicas, químicas y biológicas diferentes a las que poseía aguas arriba del mismo. En particular se ha modificado un parámetro de gran importancia en la dinámica de las comunidades fluviales y en la calidad del agua en los embalses: la temperatura.
La gran inercia térmica que presenta un embalse provoca que aguas abajo el río altere substancialmente su comportamiento térmico. Así por ejemplo, en verano el agua que sale del embalse, situado en una región de clima mediterráneo, puede presentar una temperatura notablemente inferior a la que tiene el río aguas arriba del mismo. Por tal motivo, aguas abajo de la presa el río incrementa la temperatura a lo largo de su circulación. Este incremento tiende 
a restablecer el equilibrio térmico entre el río y su entorno (atmósfera y cauce) alterado por el embalse.

Este fenómeno se presenta en el río Ebro, tomando especial relieve la gran influencia que el sistema de embalses existente aguas arriba de la Central Nuclear de Ascó (sistema Mequinenza Ribarroja - Flix) tiene en la temperatura del río. Esta influencia, más acusada en unas estaciones que en otras, condiciona fuertemente el equilibrio térmico del río. A lo largo de la presente comunicación se presentaran datos y reflexiones en este sentido.

\section{DESCRIPCIÓN DE LA ZONA EN ESTUDIO}

La Central Nuclear de Ascó se asienta en la margen derecha del río Ebro, a la altura de la po- blación que le da nombre, cinco kilómetros aguas abajo de la central hidroeléctrica de Flix.

El río tiene un importante núcleo de regulación exactamente en la zona aguas arriba de la C. N. Ascó; así, si bien la central de Flix cuenta con una reducida capacidad de embalse (del orden de $11 \mathrm{hm}^{3}$ ), inmediatamente aguas arriba se encuentra la central de Ribarroja, con una capacidad de embalse de $210 \mathrm{hm}^{3}$ y en su cola se encuentra el embalse de Mequinenza, con $1534 \mathrm{hm}^{3}$ de capacidad y verdadero centro de regulación del tramo bajo del río Ebro (figura 1). La media anual de la aportación total (Ebro más Segre y Cinca) en Ribarroja es de $18018 \mathrm{hm}^{3}$.

Teniendo en cuenta los volúmenes de embalse y las aportaciones naturales, observamos que el tiempo de residencia medio del agua será prácticamente despreciable en Flix y mucho mayor en Mequinenza.

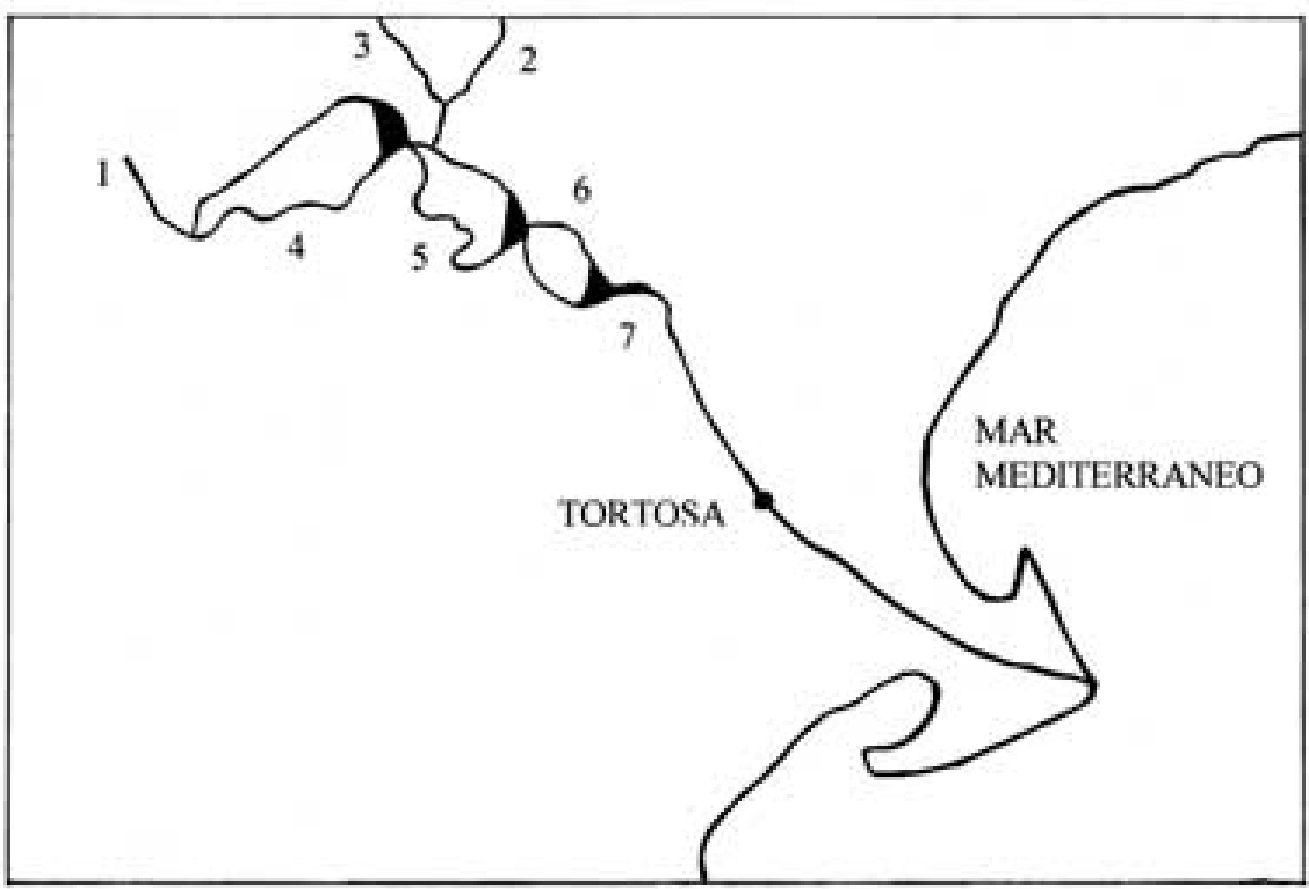

Figura 1. Localización de la zona en estudio. Aportación natural de los ríos y capacidad de almacenamiento de los embalses, (1) Río

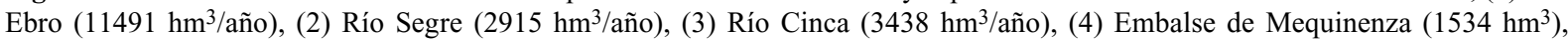
(5) Embalse de Ribarroja $\left(210 \mathrm{hm}^{3}\right)$ (6) Embalse de Flix $\left(11 \mathrm{hm}^{3}\right)$. La longitud del río estudiado(7) tiene $5.3 \mathrm{~km}$. Location of the study area. Annual flow of the rivers and reservoirs' volume capacity: (1) Ebro River (11 $\left.491 \mathrm{hm}^{3} / \mathrm{y}\right)$, (2) Segre River (2915 hm $\left.3 / \mathrm{y}\right)$,

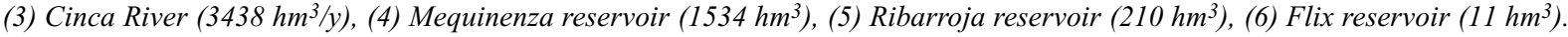
The length of the studied river (7) is $5.3 \mathrm{~km}$. 


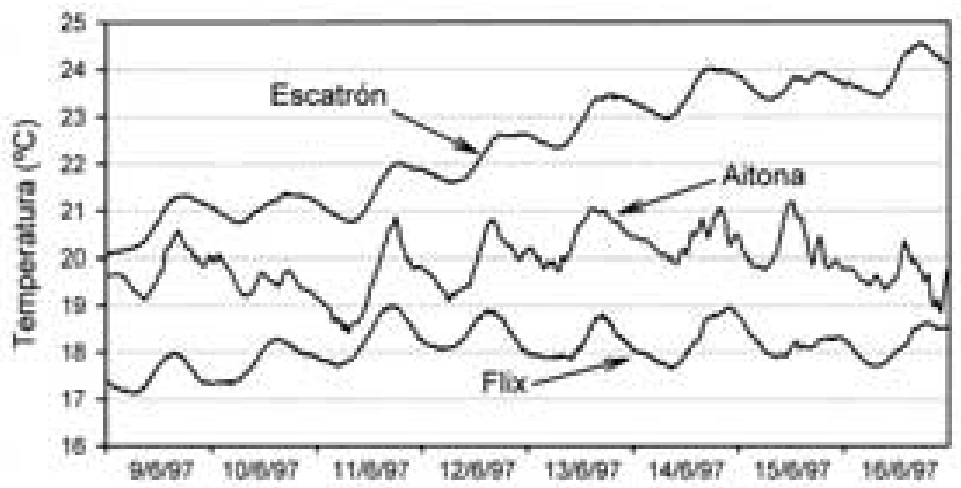

Figura 2. Temperatura del agua en Escatrón (1), Flix (2) y Aitona (3). Water temperature at Escatrón (1), Flix (2) and Aitana (3).

\section{COMPORTAMIENTO TÉRMICO DEL RÍO EBRO EN LA ZONA DE ESTUDIO}

Las capas superficiales de un embalse, en contacto con la atmósfera, responden rápidamente a estímulos externos tales como variación de la temperatura ambiente o un aumento o disminución de la insolación en mayor medida que las capas inferiores, para las que el agua que se encuentra situada encima ejerza un efecto tamponador. Así pues, cabe esperar que en las capas inferiores la variación de temperatura con el tiempo sea inferior; con lo cual, se puede producir una estratificación térmica.

En verano, la temperatura del agua de los ríos Ebro, Segre y Cinca (registros de Escatrón, Aitona y Fraga respectivamente) antes de entrar en el sistema de embalses MequinenzaRibarroja- Flix es más elevada que la temperatura del agua que sale de dicho sistema de embalses (registro en Flix). En la figura 2 se presentan las temperaturas del agua en Escatrón (que se encuentra localizado en la cola del embalse de Mequinenza), Aitona (que esta situada en la cola del embalse de Ribarroja) y de Flix (aguas abajo de la hidroeléctrica del mismo nombre); siendo posible observar dicho fenómeno. Este comportamiento puede explicarse por la existencia del sistema de embalses y la gran inercia térmica que éste ofrece al cambio de temperatura del agua.

$\mathrm{Al}$ inicio del verano, el normal aumento de la temperatura del agua en los ríos Ebro, Segre y Cinca, no es acusado de forma significativa e inmediata por el agua de los embalses debido a la gran masa de agua que en ellos es almacenada. Sin embargo, esto se ve más acusado a medida que avanza el verano, como puede observarse en la figura 3.

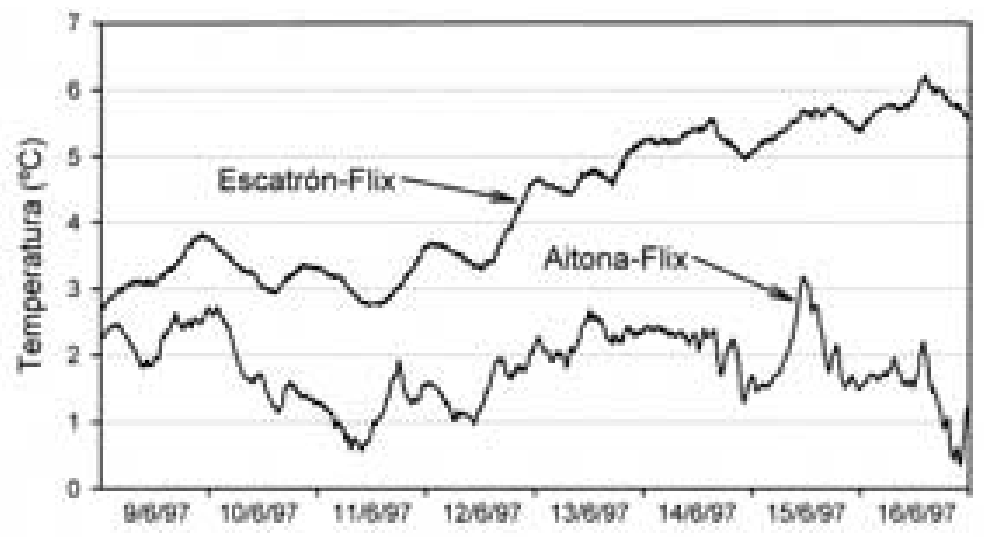

Figura 3. Diferencia de temperatura del agua entre Escatrón y Flix (1) y entre Aitona y Flix (2). Water temperature differences between Escatrón and Flix (1), and between Aitona and Flix (2). 
En la figura 4 se presentan las temperaturas, de unos días del mes de julio para las estaciones de Mequinenza, Ribarroja y Flix, tomadas a la salida de dichos embalses. La menor temperatura se produce a la salida de Mequinenza, provocado por su gran masa de agua y la lenta respuesta a los estímulos térmicos externos. Ribarroja, presenta una temperatura mayor que la de Mequinenza y menor a la de Flix; esto debido a que su embalse es de menor tamaño y la recuperación de temperatura que el agua llega a tener durante su recorrido por el mismo. A la salida de Flix se observa una mayor temperatura que en las otras dos y menor a la de las entradas, esto es por causa de su pequeño embalse con que cuenta esta hidroeléctrica (prácticamente se puede considerar como un tramo del río), por lo que se tiene una respuesta a un estimulo externo con el medio ambiente más rápida.

La estación de C. N. Asco se encuentra 5.3 kilómetros aguas abajo de la presa de Flix. En la figura 5, los datos de temperatura son también del mes de julio y en ella se comparan los de Flix y de ASCO. Debido al desequilibrio térmico que presenta el río Ebro aguas abajo de Flix con respecto a sus condiciones medioambientales, se produce un incremento en la temperatura del agua al circular ésta entre Flix y $\mathrm{C}$. N. Ascó. Este incremento llega a superar $1^{\circ} \mathrm{C}$ en las horas centrales del día (horas de mayor radiación solar incidente).

\section{INSTALACIÓN}

Las mediciones de temperatura, tanto a la salida de los embalses de Ribarroja y Flix; así como en los tres puntos de inicio y final del estudio, Escatrón, Aitona y Ascó respectivamente, se inician en Marzo de 1997; los puntos de Mequinenza y Fraga inician sus muestreos en julio. Las mediciones que se toman son cada 1 minuto y se guarda en el registro la media de cada 10 minutos; además, se registra la máxima y mínima temperatura que se presenta durante ese día.

También se registran otros parámetros necesarios para poder realizar el balance térmico. En resumen, se crearon las estaciones de medición en los puntos siguientes: Escatrón, Ribarroja, Mequinenza, Aitona, Fraga, Flix y Ascó. En los dos primeros se midieron: temperatura ambiente, radiación solar, radiación neta (incidente - reflejada), humedad relativa, velocidad del viento, dirección del viento, pluviometría, temperatura del agua, nivel del agua. Mientras que en los demás tan solo se estuvieron midiendo temperatura del agua y nivel del agua.

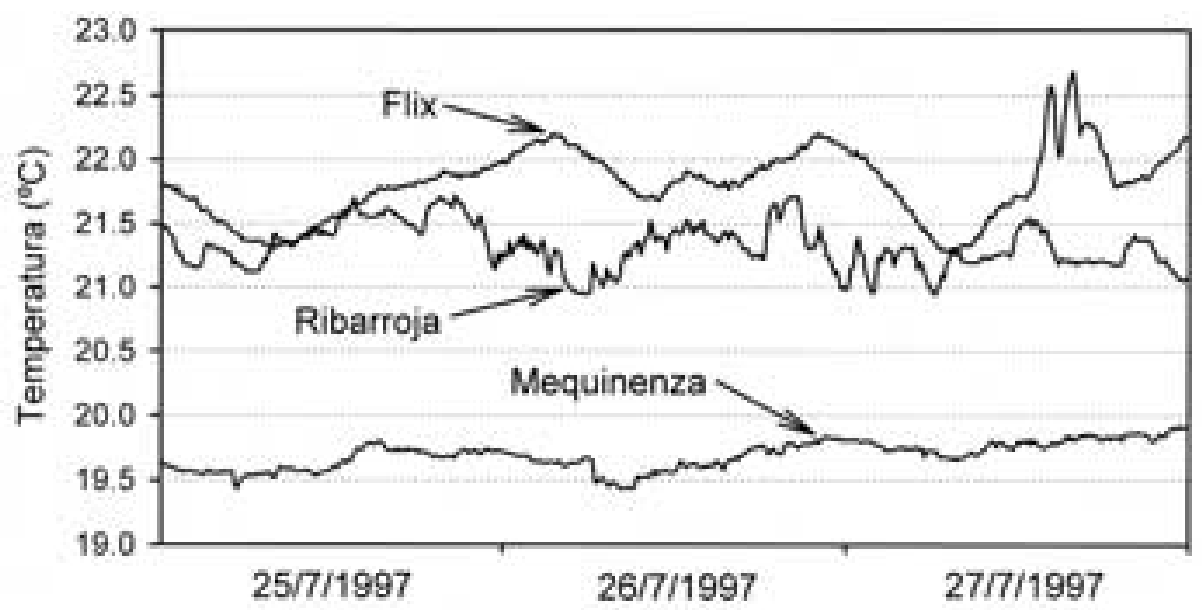

Figura 4. Temperatura del agua a la salida de los embalses de Ribarroja (1), Flix (2) y Mequinenza (3). Water temperature at the reservoir outlets of Ribarroja (1), Flix (2), and Mequinenza (3). 
BALANCE TÉRMICO EN EL RÍO AGUAS ABAJO DE LOS EMBALSES

\section{Planteamiento teórico}

Como ya se ha mencionado, el río Ebro se ve alterado en su equilibrio térmico debido a los embalses de Mequinenza, Ribarroja y Flix, provocando que en ellos la temperatura del agua en verano sea menor que la que se tiene a la entrada de Mequinenza; sin embargo, se observa una recuperación de esta temperatura a partir del ultimo embalse (Flix). Así, es posible llevar a cabo un balance térmico aguas abajo de los embalses.

Para la evaluación del calor que el agua del río absorbe de modo natural en un tramo determinado y la correspondiente variación de temperatura, se debe establecer un balance entre la energía recibida y emitida por el agua a lo largo de dicho tramo. Esto se puede llevar a cabo por medio de la metodología desarrollada por Edinger y Geyer (1974). Esta puede ser expresada como:

$$
\mathrm{A}=\mathrm{R}+\mathrm{OL}_{\mathrm{i}}-\mathrm{OL}_{\mathrm{e}}-\mathrm{H}_{\mathrm{e}}-\mathrm{H}_{\mathrm{c}}+\mathrm{S}\left(\mathrm{W} / \mathrm{m}^{2}\right)
$$

donde: A es la potencia calórica absorbida por el agua por $\mathrm{m}^{2}$ de superficie libre, $\mathrm{R}$ es la radiación solar total (directa más difusa) neta (incidente menos reflejada) de onda corta; potencia absorbida por el agua por $\mathrm{m}^{2} \mathrm{de}$ superficie libre, $\mathrm{OL}_{\mathrm{i}}$ es la radiación neta (incidente menos reflejada) de onda larga; potencia absorbida por el agua por $\mathrm{m}^{2}$ de superficie libre, $\mathrm{OL}_{\mathrm{e}}$ es la radiación de onda larga emitida por el agua del río; potencia cedida por $\mathrm{m}^{2}$ de superficie libre, $\mathrm{H}_{\mathrm{e}}$ es el calor latente de evaporación; potencia cedida por el agua por $\mathrm{m}^{2}$ de superficie libre, $\mathrm{H}_{\mathrm{c}}$ es el calor sensible de conducción atmósfera-agua; potencia cedida por $\mathrm{m}^{2}$ de superficie libre, $\mathrm{S}$ es el calor intercambiado con el substrato (lecho); potencia absorbida por el agua por $\mathrm{m}^{2}$ de cauce. Se supone que dada la poca profundidad y gran anchura del río, el ancho del cauce es sensiblemente igual al ancho de la superficie libre.

\section{Almacenamiento de calor por el agua que circula en el río}

Un cierto volumen de agua limitado por dos secciones de área $\mathrm{E}$, ancho $\mathrm{B}$ y separadas una distancia d, transportado por el río a lo largo de un tramo de longitud L y con velocidad v, durante su viaje almacena un calor igual a:

$$
A B d\left(\frac{L}{v}\right)
$$

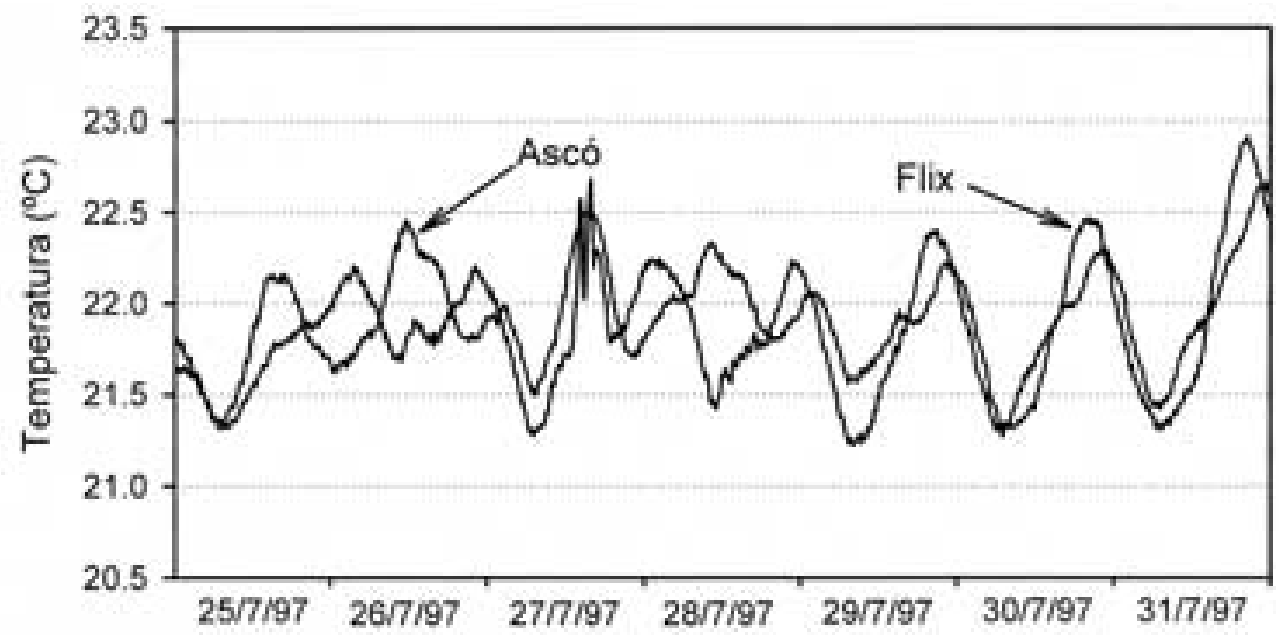

Figura 5. Temperatura del agua en Flix (1) y Ascó (2). Water temperature in Flix (1), and Ascó (2). 
deberá verificarse la igualdad:

$$
A B d\left(\frac{L}{v}\right)=\Delta T d E \rho C_{e}
$$

de donde:

$$
A=\frac{\Delta T \rho E v C_{e}}{L B}=\frac{\Delta T \rho Q C_{e}}{L B}
$$

donde $\Delta \mathrm{T}$ es el incremento de temperatura del agua en su viaje a lo largo del tramo $\left({ }^{\circ} \mathrm{C}\right), \mathrm{Q}$ es el caudal circulante $\left(\mathrm{m}^{3} / \mathrm{s}\right), \mathrm{C}_{\mathrm{e}}$ es el calor específico del agua $\left(1 \mathrm{Kcal} /{ }^{\circ} \mathrm{CKg}\right), \rho$ es la densidad del agua $\left(1000 \mathrm{~kg} / \mathrm{m}^{3}\right)$, L es la longitud del tramo estudiado (m), B es el ancho eficaz del río (m) es el ancho asociado al agua en movimiento y no incluye el agua inmovilizada junto a las márgenes.

\section{Radiación solar total neta de onda corta}

El valor de la radiación solar total neta de onda corta sobre la superficie del río, diferencia entre la radiación incidente y la reflejada. Esta ha sido medida directamente.

\section{Radiación neta de onda larga absorbida}

La radiación atmosférica incidente de onda larga se calcula mediante la ley de StefanBoltzmann. Tomando en cuenta los valores de los parámetros físicos involucrados, esto se expresa como:

$$
\mathrm{OL}_{\mathrm{i}}=5.1410^{-13}\left(\mathrm{~T}_{\mathrm{A}}\right)^{6}\left(\mathrm{~W} / \mathrm{m}^{2}\right)
$$

donde $\mathrm{T}_{\mathrm{A}}$ es la temperatura del aire $(\mathrm{K})$, medida directamente.

\section{Radiación de onda larga emitida}

La radiación de onda larga emitida a la atmósfera por el río puede asimismo evaluarse con la ley de Stefan-Boltzmann:

$$
\mathrm{OL}_{\mathrm{e}}=5.4910^{-8}\left(\mathrm{~T}_{\mathrm{s}}\right)^{4}\left(\mathrm{~W} / \mathrm{m}^{2}\right)
$$

donde $\mathrm{T}_{\mathrm{s}}$ es la temperatura de la superficie del agua $(\mathrm{K})$, medida directamente.

\section{Pérdida de calor por evaporación}

Tomando en cuenta la expresión obtenida por Seco y Garmendia (1973)

$$
\mathrm{H}_{\mathrm{e}}=1.49\left(\mathrm{e}_{\mathrm{s}}-\mathrm{e}_{\mathrm{d}}\right) \mathrm{U}^{0.76}\left(\mathrm{~W} / \mathrm{m}^{2}\right)
$$

donde $\mathrm{e}_{\mathrm{s}}$ es la tensión de vapor de saturación del aire a la temperatura del agua $(\mathrm{mm} \mathrm{Hg}), \mathrm{e}_{\mathrm{d}}$ es la tensión de vapor del aire ( $\mathrm{mm} \mathrm{Hg}), \mathrm{U}$ es la velocidad del viento $(\mathrm{m} / \mathrm{s})$, medida directamente.

\section{Pérdida de calor por conducción}

El calor sensible intercambiado por conducción entre el agua y el aire se obtiene mediante la relación de Bowen, entre el calor cedido por conducción y el calor latente de evaporación

$$
\mathrm{H}_{\mathrm{c}}=0.6917\left(\mathrm{~T}_{\mathrm{A}}-\mathrm{T}_{\mathrm{s}}\right) \mathrm{U}^{0.76}\left(\mathrm{~W} / \mathrm{m}^{2}\right)
$$

\section{Calor intercambiado con el lecho}

El agua también intercambia calor con el lecho. La importancia de este supuesto intercambio de calor ha sido puntualizada por Fristchen y Van Bavel (1963). Este intercambio consiste en almacenar el calor en las horas de fuerte radiación solar y cederlo al agua durante la noche. El flujo de calor entre el agua y el lecho afecta a los estratos que se encuentran situados por encima de una cierta profundidad.

Si consideramos el aporte neto diario del substrato, al ser las temperaturas del río sensiblemente cíclicas a lo largo del día, cabe esperar que tienda anularse. Los aportes horarios, no obstante, no son despreciables, y si se realiza un balance energético con esta frecuencia de muestreo, deben ser tenidos en cuenta.

\section{Ajuste de parámetros en el balance energético}

La ecuación de almacenamiento de calor, descrita anteriormente, esta dada como

$$
A=\frac{\Delta T \rho Q C_{e}}{L B}
$$


Agrupando los términos que son conocidos, se tiene que

$$
A=\frac{\Delta T \rho Q C_{e}}{L}
$$

Así, es posible que la ecuación de almacenamiento de calor este en función del ancho efectivo del río, B; de tal forma se tiene que

$$
A=\frac{K}{B}
$$

Por otra parte, se sabe que el balance de energía esta dado por

$$
\mathrm{A}=\mathrm{R}+\mathrm{OL}_{\mathrm{i}}-\mathrm{OL}_{\mathrm{e}}-\mathrm{H}_{\mathrm{e}}-\mathrm{H}_{\mathrm{c}}+\mathrm{S}\left(\mathrm{W} / \mathrm{m}^{2}\right)
$$

de la cual, casi todos los valores son conocidos a excepción del calor intercambiado con el lecho, S; de ahí que

$$
\mathrm{A}=\mathrm{C}+\mathrm{S}\left(\mathrm{W} / \mathrm{m}^{2}\right)
$$

donde

$$
\mathrm{C}=\mathrm{R}+\mathrm{OL}_{\mathrm{i}}-\mathrm{OL}_{\mathrm{e}}-\mathrm{H}_{\mathrm{e}}-\mathrm{H}_{\mathrm{c}}\left(\mathrm{W} / \mathrm{m}^{2}\right)
$$

Así, dadas un conjunto de medidas con frecuencias horarias, se puede establecer la ecuación del balance energético, teniendo así un conjunto de expresiones del tipo

$$
\frac{K_{i}}{B}=C_{i}+S_{i} \quad \mathrm{i}=1 \ldots 24
$$

donde: $\mathrm{K}_{\mathrm{i}}$ es el valor conocido para cada hora, $\mathrm{B}$ es el ancho eficaz del río $\mathrm{C}_{\mathrm{i}}$ es el parámetro conocido.

$\mathrm{S}_{\mathrm{i}}$ es el calor intercambiado con el lecho, $1 . .24$ representa el conjunto de medidas que cubren un día completo, en el que, como se ha comentado, la suma de todos los $\mathrm{S}_{\mathrm{i}}$ debe ser nula

$$
\sum_{i=1}^{24} S_{i}=0
$$

Realizando el balance energético a lo largo de un día completo, debe verificarse

$$
\sum_{i=1}^{24} \frac{K_{i}}{B}=\sum_{i=1}^{24} C_{i}
$$

por lo que

$$
B=\sum_{i=1}^{24} K_{i} / \sum_{1}^{24} C_{i}
$$

permite ajustar el ancho eficaz del río, con independencia de $\mathrm{S}_{\mathrm{i}}$. Este procedimiento es válido para series de 24,48 , etc. y en general un número de horas tal que comprenda un número entero de días.

Conocido el valor de $\mathrm{B}$, se tiene

$$
S_{i}=\frac{K_{i}}{B} * C_{i}
$$

\section{RESULTADOS}

Con anterioridad al estudio que actualmente se esta realizando, se llevo a cabo un primer balan-

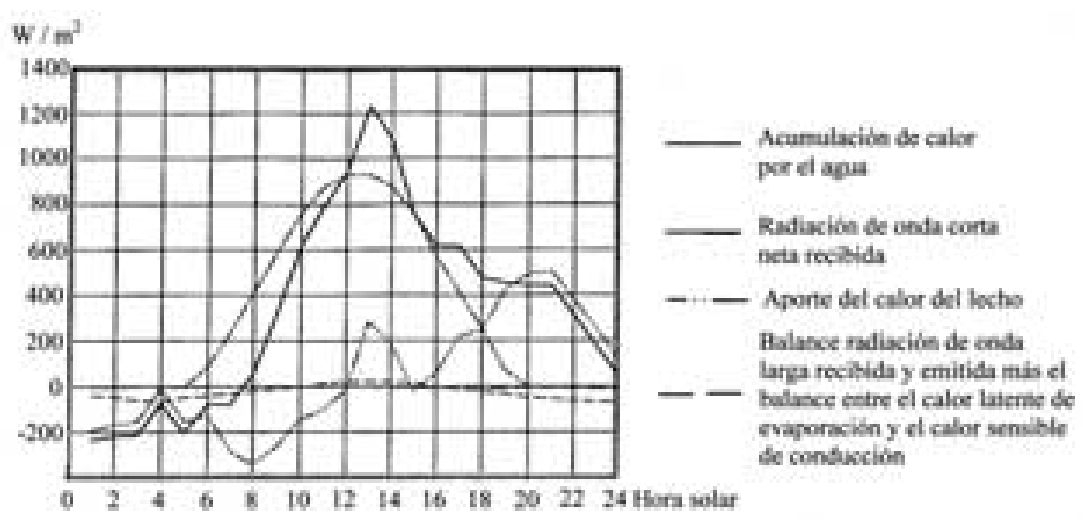

Figura 6. Balance energético a lo largo de un día. Daily energy budget. 
ce energético en un tramo de $5.3 \mathrm{~km}$. entre Flix y ASCO con un conjunto de datos correspondientes al mes de agosto de 1990.

Al aplicar la ecuación de balance de acuerdo con lo expuesto anteriormente, fue posible tener unos primeros resultados; de los cuales se obtiene que el ancho eficaz del río vale $B=120 \mathrm{~m}$. Teniendo presente que de los planos topográficos $(1: 5000)$ se valoraba en $140 \mathrm{~m}$; sin embargo, en esta medida se tomaba en cuenta la zona de agua inmovilizada junto a las márgenes.

En la figura 6, se muestra el resultado del balance energético de uno de los días para este periodo. En él, se refleja la acumulación de calor de agua (A), la radiación de onda corta neta recibida (R), el aporte del calor del lecho (S), el balance de radiación de onda larga recibida y emitida $\left(\mathrm{OL}_{\mathrm{i}}-\mathrm{OL}_{\mathrm{e}}\right)$ y el balance entre el calor latente de evaporación y el calor sensible de conducción $\left(\mathrm{H}_{\mathrm{e}}-\mathrm{H}_{\mathrm{c}}\right)$.

En la gráfica se puede observar que el valor de la radiación de onda corta es quien juega uno de los papeles importantes en el balance global junto con los términos de acumulación de calor por el agua y de incremento de calor con el lecho. Los balances de onda larga y de evaporación-conducción prácticamente no tienen incidencia.

La aportación neta diaria del lecho es prácticamente nula, como ya se había comentado en el planteamiento teórico; por lo que el calor acumulado por el agua es casi igual al recibido por la radiación de onda corta. Sin embargo, el lecho juega un papel regulador, de modo que absorbe calor cuando el agua tiende a calentarse (horas centrales del día) y cederlo cuando tiende a enfriarse (durante la noche).

\section{CONCLUSIONES}

A continuación se presentan las conclusiones más importantes de este estudio:

1. Como se ha visto, en verano y en clima mediterráneo, los datos reportan un enfria- miento del agua por efecto de los embalses; lo que condiciona la vida en las comunidades fluviales.

2. El agua que circula aguas abajo del embalse, tiende a recuperar la temperatura de equilibrio térmico de acuerdo con las condiciones ambientales.

3. Aplicando la ecuación del balance energético a un tramo de $5.3 \mathrm{~km}$. situado inmediatamente aguas abajo del sistema de embalses Mequinenza-Ribarroja-Flix en el río Ebro (España), para la época de verano, fue posible observar que las variables más importantes son la radiación solar total neta de onda corta, almacenamiento de calor y el calor intercambiado con el lecho.

\section{AGRADECIMIENTOS}

El presente estudio se realiza dentro de los proyectos de investigación REN 2001-2185-CO2$\mathrm{O} 1$ y $\mathrm{O} 2$ financiados por el Programa de Recursos Hídricos del Plan Nacional de Investigación y Desarrollo. Agradecemos al Instituto de Cooperación Iberoamericano la beca que concedida a Rafael Val Segura para poder llevar a cabo el doctorado en la Universidad Politécnica de Cataluña, Barcelona.

\section{REFERENCIAS}

EDINGER, J. E.\& D. K. BRADY \& J. C. GEYER. 1974. Heat Exchange and Transport in the Environment, Rep. 14, Electric Power Research Institute, Palo Alto, California.

FRITSCHEN, L. J. \& C. H. M. VAN BAVEL, 1963: Evaporation from Shallow Water and Related Micrometeorological Parameters. Journal of Applied Meteorology, 2(3): 407-411.

DOLZ, J. y J. PUERTAS. 1990. Estudio del Comportamiento del Río Ebro en Verano. Universidad Politécnica de Cataluña, Barcelona, España. $124 \mathrm{pp}$

SECO, J \& J. GARMENDIA. (1973). Evaporación en España. Instituto Nacional de Meteorología, Publicación A-52, Madrid, España. 30 pp 